\title{
Petunia- and Arabidopsis-Specific Root Microbiota Responses to Phosphate Supplementation
}

\author{
Natacha Bodenhausen, ${ }^{1,2}$ Vincent Somerville, ${ }^{1}$ Alessandro Desirò, ${ }^{3}$ Jean-Claude Walser, ${ }^{4}$ Lorenzo Borghi, ${ }^{5}$ \\ Marcel G. A. van der Heijden, ${ }^{1,6,7}$ and Klaus Schlaeppi ${ }^{1,8, \dagger}$ \\ ${ }^{1}$ Division of Agroecology and Environment, Agroscope, Zurich, Switzerland \\ ${ }^{2}$ Department of Soil Sciences, Research Institute of Organic Agriculture FiBL, Frick, Switzerland \\ ${ }^{3}$ Department of Plant, Soil and Microbial Sciences, Michigan State University, East Lansing, MI, U.S.A. \\ ${ }^{4}$ Genetic Diversity Centre, ETH Zurich, Zurich, Switzerland \\ ${ }^{5}$ Institute of Plant and Microbial Biology, University of Zurich, 8008 Zurich, Switzerland \\ ${ }^{6}$ Institute for Evolutionary Biology and Environmental Studies, University of Zurich, Zurich, Switzerland \\ ${ }^{7}$ Plant-Microbe Interactions, Institute of Environmental Biology, Faculty of Science, Utrecht University, Utrecht, The Netherlands \\ ${ }^{8}$ Institute of Plant Sciences, University of Bern, Switzerland
}

Accepted for publication 27 March 2019.

\section{ABSTRACT}

Phosphorus $(P)$ is a limiting element for plant growth. Several root microbes, including arbuscular mycorrhizal fungi (AMF), have the capacity to improve plant nutrition and their abundance is known to depend on $\mathrm{P}$ fertility. However, how complex root-associated bacterial and fungal communities respond to various levels of $P$ supplementation remains ill-defined. Here we investigated the responses of the root-associated bacteria and fungi to varying levels of $\mathrm{P}$ supply using $16 \mathrm{~S}$ rRNA gene and internal transcribed spacer amplicon sequencing. We grew Petunia, which forms symbiosis with AMF, and the nonmycorrhizal model species Arabidopsis as a control in a soil that is limiting in plant-available $\mathrm{P}$ and we then supplemented the plants with complete fertilizer solutions that varied only in their phosphate concentrations. We searched for microbes, whose abundances varied by $\mathrm{P}$ fertilization, tested whether a core microbiota responding to the $P$ treatments could be identified and asked whether bacterial and fungal co-occurrence patterns change in response to the varying $\mathrm{P}$ levels. Root microbiota composition varied substantially in response to the varying $\mathrm{P}$ application. A core microbiota was not identified as different bacterial and fungal groups responded to low-P conditions in Arabidopsis and Petunia. Microbes with $\mathrm{P}$-dependent abundance patterns included Mortierellomycotina in Arabidopsis, while in Petunia, they included AMF and their symbiotic endobacteria. Of note, the P-dependent root colonization by AMF was reliably quantified by sequencing. The fact that the root microbiotas of the two plant species responded differently to low-P conditions suggests that plant species specificity would need to be considered for the eventual development of microbial products that improve plant $P$ nutrition.

Keywords: Arabidopsis thaliana, arbuscular mycorrhizal fungi, fungal endobacteria, microbiome, Petunia hybrida, phosphate, rhizosphere and phyllosphere, root microbiota
${ }^{\dagger}$ Corresponding author: K. Schlaeppi; klaus.schlaeppi@ips.unibe.ch

Author Contributions: N.B., M.v.d.H., and K.S. designed the experiments. N.B. performed the experiment, did the molecular work and analyzed the data. J.C.W., V.S., A.D., and K.S. contributed to data analysis. N.B., L.B., M.v.d.H., and K.S. wrote the manuscript. All authors approved the final manuscript.

Funding: This study was supported by the Scientific Education and Research Institute (grant C14.0132 to M.v.d.H. and K.S.) and by the Gebert Rüf Foundation (grant GRS-072/17 to N.B., M.v.d.H., and K.S.)

*The $\boldsymbol{e}$-Xtra logo stands for "electronic extra" and indicates that 10 supplementary figures, five supplementary tables, and five supplementary data files are published online.

The author(s) declare no conflict of interest.
Phosphorus $(\mathrm{P})$ presents one of the key nutrients for plant growth. While a small fraction of soil $\mathrm{P}$, the inorganic $\mathrm{P}$ (orthophosphate) is directly available for plant uptake, the larger fraction is complexed to organic and mineral soil components and therefore inaccessible for plants. The conventional agronomic solution to increase $\mathrm{P}$ availability for plants relies on supplementing mineral phosphate $\left(\mathrm{PO}_{4}{ }^{3-}\right)$. However, yield optimization requires excess application of phosphate since less than $30 \%$ of applied P fertilizers effectively support plant growth, the rest of the applied phosphate readily transforms to plant nonavailable $P$ forms (Cordell et al. 2009). Such an overuse causes the rapid depletion of finite phosphorus reservoirs, is expensive and causes environmental harm, primarily with negative impacts on the aquatic environment by eutrophication of the surface water (Cordell et al. 2009; Reijnders 2014; Scholz and Wellmer 2013). Therefore, agriculture searches for novel sustainable 
solutions that reduce fertilizer inputs and increase the nutrient-use efficiency while maintaining high plant yields.

Numerous root-associated microbes have the capacity to mobilize soil P or metabolize recalcitrant forms (Gyaneshwar et al. 2002; Jacoby et al. 2017), thereby enhancing plant performance and agricultural yield especially under nutrient limiting conditions. For fungi, there is a continuum of functionally similar associations between different groups of root fungi and their host plant species (van der Heijden et al. 2017). For instance, arbuscular mycorrhizal are intimately connected to plant roots and support plant growth by mobilizing and transporting $\mathrm{P}$ from a larger soil volume and more distant pools of $\mathrm{P}$ thanks to their large hyphal network (Jakobsen et al. 1992). Aside from these classical mycorrhizal plants, also nonmycorrhizal plants such as Arabidopsis thaliana (hereafter: Arabidopsis) and Arabis alpina rely on fungal associations for nutrient acquisition. They rely on beneficial fungal endophytes including Colletotrichum tofieldiae (Hiruma et al. 2016), Serendipita indica (formerly Piriformospora indica) (Yadav et al. 2010) or fungi of the order Helotiales (Almario et al. 2017). Typically, the $\mathrm{P}$ availability in soil determines to which extent a plant is colonized by the fungal symbiotic partner with high levels of colonization under low-P conditions and little colonization in soils with high-P levels (Smith and Read, 2008). In addition to fungi, many root bacteria can support plant nutrition with their abilities to solubilize inorganic $\mathrm{P}$ or to mineralize organic $\mathrm{P}$ (Alori et al. 2017; Rodŕguez and Fraga 1999). Although their agronomic relevance remains to be proven, powerful $\mathrm{P}$ solubilizing bacteria have been identified using in vitro assays and they include strains from the genera Bacillus, Pseudomonas, and Rhizobium; for a more comprehensive list as well as their growth effects on crops, we refer to Alori et al. (2017). While a wide range of individual rhizosphere microbes is known to support plant $\mathrm{P}$ nutrition, the effects of $\mathrm{P}$ availability on the overall root microbiota remains less understood. A deeper understanding of interactions between plants and their microbial allies in response to the bioavailability of $\mathrm{P}$ is needed for developing microbe-dependent $\mathrm{P}$ fertilization solutions (Busby et al. 2017; Schlaeppi and Bulgarelli 2015).

Interactions among microbes emerge as a critical component for the maintenance of host-microbial homeostasis and for plant performance (Hassani et al. 2018). Interkingdom microbial associations occur in the plant root microbiota as for instance root fungi hosting endobacteria in their cells (Desirò et al. 2014). Such ancient fungi-endobacteria interactions (Bonfante and Desirò 2017) include root fungi of Mucoromycota (Spatafora et al. 2017) that host diverse bacterial endosymbionts related to Burkholderia and/or Mycoplasma. An example of Burkholderia-related endobacteria includes 'Candidatus Glomeribacter gigasporarum' that is hosted by species of the Gigasporaceae (family of Glomeromycotina) (Bianciotto et al. 2003). The occurrence and functional contribution of fungal endobacteria adds a further level of complexity to the interactions of plant with their associated microbes.

Plants are more and more recognized in context with their microbial communities, where a multitude of microbes collectively function as a microbiome. $\mathrm{P}$ fertilization as well as $\mathrm{P}$ depletion are known to induce shifts in soil microbial communities (Bergkemper et al. 2016; Huang et al. 2016; Ikoyi et al. 2018; Leff et al. 2015; Wakelin et al. 2012). For example, grassland soil microbes consistently responded to phosphate inputs with compositional community changes, as for instance mycorrhizal fungi, oligotrophic bacteria, and methanogenic Archaea decreased in relative abundance with nutrient additions (Leff et al. 2015). Although the responses of root-associated microbial communities to varying levels or different sources of $\mathrm{P}$ have been studied for several plant species (Almario et al. 2017; Fabiańska et al. 2018; Robbins et al. 2018;
Silva et al. 2017), comparisons between mycorrhizal and nonmycorrhizal plants have received less attention. For instance, soil $\mathrm{P}$ levels presented a major driver for the root and rhizosphere microbiota structure as determined for mycorrhizal maize grown in the field comparing low- and high-P availability (Gomes et al. 2018). Roots from low-P conditions contained abundant slow-growing bacterial taxa such as Burkholderiaceae while fastgrowing and copiotrophic Enterobacteriaceae and Pseudomonadaceae were enriched in high-P conditions. Consistent with the expectation, the low-P soil had a positive effect on the abundance of arbuscular mycorrhizal fungi (AMF). Robbins et al. (2018) investigated the effects of different levels of soil $\mathrm{P}$ availability on the rhizosphere and root microbiota of nonmycorrhizal Arabidopsis using soil from a long-term fertilization experiment. While P fertilization had little effects on microbial diversity, they affected more strongly the plant-associated microbiota compared with bulk soil communities, suggesting plant-mediated cues for structuring the plant microbiota. Indeed, Fabiańska et al. (2018) found that plant nutritional status affected the assembly of the fungal community in Arabidopsis roots. This work revealed that changes in root fungal communities were correlated with changes in the status of the plant's phosphate starvation response and that mutants in phosphate signaling network genes exhibited similarly altered root fungal communities. Work on model nonmycorrhizal plants revealed robust responses of the root microbiota to the availability of soil P. Comparative work with mycorrhizal plants, which actually present the majority of land plants, is needed to integrate arbuscular mycorrhizal symbionts into a framework of general root microbiota responses to $\mathrm{P}$ availability.

Petunia $\times$ hybrida (hereafter: Petunia) is a model plant that is commonly used for investigating the symbiosis with AMF (Breuillin et al. 2010; Wegmüller et al. 2008). Due to its fast life cycle, modest size and the availability of genetic tools made, Petunia is also a model to study plant development (Vandenbussche et al. 2016), plant nutrition (Liu et al. 2018), and hormonal signaling (Hamiaux et al. 2012). Petunia belongs to the family Solanaceae, thus is related to tomato, potato, and eggplant so that root microbiota knowledge may be transferable to these staple food crops. We therefore chose Petunia in comparison with nonmycorrhizal Arabidopsis to study root microbiota responses to varying $\mathrm{P}$ availability and how plants cope with P limiting conditions.

In this study, we mainly manipulated the phosphate levels in the fertilizer solution and tested the hypothesis that the composition of the root microbiota changes depending on $\mathrm{P}$ supply. We asked whether a core microbiota or plant species-specific microbiotas prevail in response to $\mathrm{P}$ deficiency. We expected the Petunia root microbiota to enrich for AMF under low-P conditions, whereas the Arabidopsis response to low-P remained unclear. Further, we were interested in the bacterial responses of the two plant species and whether the same or different sets of bacteria will respond to the varying levels of P. A particular goal of the study was to examine bacterial and fungal co-occurrence patterns in response to the varying $\mathrm{P}$ levels. We expected to find potential microbial interactions and hypothesized that the root microbiota data contains paired sequence information of fungal endobacteria and their corresponding host fungi. Finally, a technical goal of this study was to quantify AMF colonization in the context of whole fungal diversity based on DNA-based sequencing instead of the traditional morphological quantification by microscopy.

This study reveals that root microbiota composition varies markedly by the $\mathrm{P}$ levels in the fertilizer solution and that different fungal and bacterial groups are responsive to low-P conditions in Arabidopsis and Petunia. We find co-abundant groups of candidate microbial cooperation partners, including AMF and their symbiotic 
endobacteria, both known to support plant growth under low-P conditions. Our work suggests that Arabidopsis and Petunia have evolved individual microbial solutions, involving multitrophic microbial interactions, to cope with low-P conditions.

\section{MATERIALS AND METHODS}

Plant growth. Soil was collected on 4 April 2014 from an agricultural grassland field site $\left(47^{\circ} 26^{\prime} 20^{\prime \prime} \mathrm{N} 8^{\circ} 31^{\prime} 40^{\prime \prime} \mathrm{E}\right)$, sieved to $2 \mathrm{~mm}$ and stored at $4^{\circ} \mathrm{C}$ until use. Soil was mixed $1: 1$ by volume with sterilized quartz-sand and analyzed for chemical properties at the Labor für Boden-und Umweltanalytik (Eric Schweizer AG, Thun, Switzerland): $\mathrm{pH} 6.8,7 / 13 / 78 \%$ (clay/silt/sand) and 7.8/1.05/ $1.07 \mathrm{mg} \mathrm{kg}^{-1}$ (water-extractable $\mathrm{N} / \mathrm{P} / \mathrm{K}$ ). The sand-soil mixture presents a neutral experimental soil containing low levels of plant-available $\mathrm{N}, \mathrm{P}$, and $\mathrm{K}$. The experiments were conducted in $400 \mathrm{ml}$ pots lined with a mesh (Trenn-Vlies, Windhager, Thalgau, Germany).

Petunia (line V26) and Arabidopsis (accession Col-0) seeds were surface sterilized with $70 \%$ ethanol during $2 \mathrm{~min}$, washed with autoclaved water and plated on 1/2 strength Murashige and Skoog basal medium (Sigma, Buchs, Switzerland) supplemented with $1.5 \%$ sucrose and solidified with $1.5 \%$ agar. Plants were germinated under long-day conditions (16-h photoperiod) in climate chamber (Sanyo MLR-352H; Panasonic, Osaka, Japan) at $25^{\circ} \mathrm{C}$ and $60 \%$ relative humidity. After 7 days, seedlings were transferred to $400 \mathrm{ml}$ pots filled with the experimental sand-soil mixture. Plants $(n=7$ to 9) were grown for 2 weeks in the same climate chamber then moved to an in-house climate chamber with same humidity, photoperiod, and temperature.

Plants were fertilized with a corrected nutrient solution following (Reddy et al. 2007). We manipulated the phosphate levels in the fertilizer according to Breuillin et al. (2010) by preparing three nutrient solution (Base solution: $3 \mathrm{mM} \mathrm{MgSO}_{4}, 0.75 \mathrm{mM} \mathrm{KNO}_{3}$, $0.87 \mathrm{mM} \mathrm{KCl}, 1.52 \mathrm{mM} \mathrm{Ca}\left(\mathrm{NO}_{3}\right)_{2}, 0.02 \mathrm{mM}$ NaFe EDTA, $11 \mathrm{mM}$ $\mathrm{MnSO}_{4}, 1 \mu \mathrm{M} \mathrm{ZnSO}_{4}, 30 \mu \mathrm{M} \mathrm{H}_{3} \mathrm{BO}_{3}, 0.96 \mu \mathrm{M} \mathrm{CuSO}_{4}, 0.03 \mu \mathrm{M}$ $\left(\mathrm{NH}_{4}\right)_{6} \mathrm{Mo}_{7} \mathrm{O}_{24}$, and $\left.0.01 \mu \mathrm{M} \mathrm{Na} \mathrm{MoO}_{4}\right)$ with $\mathrm{KH}_{2} \mathrm{PO}_{4}$ concentrations of $0.03 \mathrm{mM}$ (low-P), $1 \mathrm{mM}$ (medium-P), and $5 \mathrm{mM}$ (high$\mathrm{P})$. Of note, with the varying amounts of $\mathrm{KH}_{2} \mathrm{PO}_{4}$ we manipulated the phosphate and potassium levels in the three fertilizer solutions. Nevertheless, we decided not to counterbalance the low- and medium-P solutions for their concomitant shortage of K (i) because the base solution of the fertilizer contains basic levels of $\mathrm{K}$ (sources: $\mathrm{KNO}_{3}$ and $\mathrm{KCl}$ ) and (ii) because an additional anion (from counterbalancing with a "nonphosphate K+-salt"; e.g., additional $\mathrm{KCl}$ ) would again perturb the nutrient balance. $\mathrm{KH}_{2} \mathrm{PO}_{4}$, being the sole phosphate source, establishes a strong fertilization gradient from limiting to sufficient $\mathrm{P}$ levels while the presence of basic $\mathrm{K}$ levels in the fertilizers results in a weaker K-fertilization gradient. For simplicity, we refer to the varying phosphate levels in the fertilizer throughout the manuscript but we also admit potential influence of $\mathrm{K}$ in the discussion. Each plant received $250 \mathrm{ml}$ of the solution over the last six weeks before harvest resulting in fertilized amounts of $\mathrm{P}$ corresponding to $0.7,22.8$, and $113.9 \mathrm{mg}$ of $\mathrm{P} \mathrm{kg}^{-1}$ of soil, respectively.

Sample collection. We conducted three separate experiments using the same batch of the sand-soil mixture, growth conditions and $\mathrm{P}$ fertilizer treatments. In the first experiment, we quantified shoot biomass, leaf nutrient levels and levels of AMF root colonization to establish and validate our experimental system. In the second experiment we only grew Petunia in the low-P conditions to collect root samples for comparing different internal transcribed spacer (ITS) profiling approaches. In the third experiment, we confirmed the efficacy of the fertilizer treatments by scoring shoot biomass and AMF root colonization levels and employed the plant root samples for microbiota profiling.

Plants were harvested at 10 weeks. The shoots were separated from the roots with a clean scalpel. The shoots were dried in a $60^{\circ} \mathrm{C}$ oven for dry weight and leaf nutrient analyses. The dried shoot material was milled and analyzed for $\mathrm{P}$ and $\mathrm{K}$ concentrations using inductively coupled plasma-optical emission spectroscopy (ICPOES) at the elemental analytic department of Agroscope according to (VDLUFA-Verlag 2006).

Our root microbiota sampling method does not discriminate between endophytic or rhizoplane microbes and we refer to this sampling unit as "root-associated" or simply "root" microbiota. The loosely attached soil was shaken from the roots, the roots were washed three times in phosphate-buffered saline buffer $(137 \mathrm{mM}$ $\mathrm{NaCl}, 2.7 \mathrm{mM} \mathrm{KCl}, 8 \mathrm{mM} \mathrm{Na}_{2} \mathrm{HPO}_{4}$, and $1.5 \mathrm{mM} \mathrm{KH}_{2} \mathrm{PO}_{4}$, pH 7.0; approximately $10 \mathrm{ml}$ for $1 \mathrm{~g}$ of fresh weight) and then split into two equivalent subsamples. Samples for DNA extraction and microscopy were stored at $-80^{\circ} \mathrm{C}$ and in $50 \%$ ethanol until further processing, respectively. After staining with pen ink (Vierheilig et al. 1998), root length colonization was determined using the magnified intersections method for 100 intersections per sample (McGonigle et al. 1990). Soil from unplanted pots was collected $(n=4)$ by removing the top $1 \mathrm{~cm}$ layer and then mixing the soil below, one sample ( 250 to $500 \mathrm{mg}$ ) was taken from each pot.

DNA extraction and PCR. DNA was extracted from the root and soil samples using the NucleoSpin Soil kit (Macherey-Nagel, Düren, Germany). Roots were lyophilized, placed in $2 \mathrm{ml}$ centrifuge tubes, to which one metal bead was added. Samples were ground to a fine powder for $2 \mathrm{~min}$ at $25 \mathrm{~Hz}$ using a Retsch TissueLyser (Retsch, Haan, Germany). Buffer SL1 and enhancer solution SX was used. DNA was quantified with Picogreen and diluted to $1 \mathrm{ng} / \mu \mathrm{l}$ for soil samples and $10 \mathrm{ng} / \mu \mathrm{l}$ for root samples.

We first evaluated three PCR approaches to compare the levels of coamplified plant sequences, abundance of AMF and general fungal diversity in Petunia roots: (i) ITS1F (Gardes and Bruns 1993) and ITS2 (White et al. 1990), (ii) fITS7 (Ihrmark et al. 2012) and ITS4 (White et al. 1990), and (iii) ITS1F with the reverse complement of fITS7. The Supplementary Data File S1 contain the bioinformatic script, barcode-to-sample assignments, input data, analysis script, and markdown report for the comparison of the PCR approaches.

Based on this analysis, the PCR primers ITS1F and ITS2 were chosen to study the fungal community. PCR primers 799F (Chelius and Triplett 2001) and 1193R (Bodenhausen et al. 2013) were used to amplify hypervariable regions V5, V6, and V7 of the 16S rRNA gene for the bacterial community. To confirm the fungal community results from Illumina sequencing, we prepared an additional library for SMRT sequencing where the entire ITS region was amplified with the PCR primers ITS1F and ITS4.

PCR reactions for each library were prepared in similar way. The reaction volume was $20 \mu \mathrm{l}$ and contained $1 \times 5$ Prime Hot Master mix $200 \mathrm{nM}$ of each primer and $0.3 \%$ bovine serum albumin. Cycling programs consisted of an initial denaturation at $94^{\circ} \mathrm{C}$ for $2 \mathrm{~min}$ (16S) or $3 \mathrm{~min}$ (ITS, MiSeq, and SMRT), followed by 30 cycles of denaturation at $94^{\circ} \mathrm{C}$ for $30 \mathrm{~s}(16 \mathrm{~S}), 45 \mathrm{~s}$ (ITS, MiSeq, and SMRT), annealing at $55^{\circ} \mathrm{C}\left(16 \mathrm{~S}\right.$, ITS-SMRT) or $50^{\circ} \mathrm{C}$ (ITS-MiSeq) for $30 \mathrm{~s}$ (16S) or $1 \mathrm{~min}$ (ITS, MiSeq, and SMRT), and elongation at $65^{\circ} \mathrm{C}$ $(16 \mathrm{~S})$ or $72^{\circ} \mathrm{C}$ (ITS, MiSeq, and SMRT) for $30 \mathrm{~s} \mathrm{(16S)} \mathrm{or} 1 \mathrm{~min}$ (SMRT) or $90 \mathrm{~s}$ (ITS-MiSeq). PCR were run in triplicate with a negative control for each primer mix and verified on a $1 \%$ agarose gel.

Triplicate PCR products were pooled, cleaned with PCR clean-up kit (Macherey-Nagel), quantified using a Quant-iT Picogreen dsDNA Assay Kit (Invitrogen, Eugene, OR, U.S.A.) on a Varian Cary Eclipse fluorescence spectrometer (Agilent Technologies, 
Santa Clara, CA, U.S.A.). Equimolar amount of each PCR product were combined. For $16 \mathrm{~S}$ library preparation, the smaller band which corresponds to $16 \mathrm{~S}$ rRNA gene was selected with the gel extraction kit (Macherey-Nagel). Pooled PCR products were concentrated with Agencourt AMPure XP kit (Beckman Coulter, Brea, CA, U.S.A.) and quantified with Qubit dsDNA HS assay on a Qubit 2.0 fluorometer (Invitrogen) and combined with other libraries before sequencing.

Sequencing and bioinformatics. The MiSeq libraries were prepared at the Functional Genomics Center Zurich (www.fgcz.ch) with the NEBNext DNA library Ultra kit (New England Biolabs, Ipswich, MA, U.S.A.). After end-repairing and polyadenylating the amplicons, NEBNext Adaptor were ligated. The ligated samples were run on a $2 \%$ agarose gel and the desired fragment length were excised (50 bp \pm the target fragment length). DNA from the gel was purified with MinElute Gel Extraction Kit (Qiagen, Hilden, Germany). Fragments containing Nebnext adapters on both ends were selectively enriched with PCR using four cycles. Quality and quantity of the enriched libraries were validated using Qubit (1.0) Fluorometer and Tapestation (Agilent Technologies). The libraries were normalized to $4 \mathrm{nM}$ in Tris-Cl $10 \mathrm{mM}, \mathrm{pH} 8.5$, with $0.1 \%$ Tween 20. The libraries were sequenced at FGCZ on the Illumina MiSeq Personal Sequencer (Illumina, San Diego, CA, U.S.A.) using a 600 cycle v3 Sequencing kit (Cat. no. MS-102-3003), in paired-end $2 \times 300$ bp mode.

The PacBio SMRT bell library was prepared at the FGCZ using the DNA Template Prep Kit 1.0 (Pacific Biosciences p/n 100-259100), following the manufacturer's instructions. After DNA quantification with a Qubit Fluorometer dsDNA Broad Range assay (Life Technologies p/n 32850), the fragment size distribution was assessed with a Bioanalyzer $210012 \mathrm{~K}$ DNA Chip assay (Agilent $\mathrm{p} / \mathrm{n}$ 5067-1508). A total of 500 to $750 \mathrm{ng}$ of amplicon DNA was DNA-damage repaired and end-repaired using polishing enzymes. A blunt end ligation reaction followed by exonuclease treatment was performed to create the SMRT bell template. The library was quality inspected and quantified on the Bioanalyzer $12 \mathrm{~kb}$ DNA Chip and on a Qubit Fluorometer (Life technologies), respectively. A ready to sequence SMRT bell-Polymerase Complex was created using the Sequel binding kit 2.0 (Pacific Biosciences p/n 100-862200) according to the manufacturer instructions. The Pacific Biosciences Sequel instrument was programmed to sequence the sample on 1 Sequel SMRT Cell 1M v2 (Pacific Biosciences p/n 101-008-000), taking 1 movie of $10 \mathrm{~h}$ using the Sequel Sequencing Kit 2.0 (Pacific Biosciences p/n 101-310-400). After the run, the quality of the sequencing data were checked using the 'run QC module' of the PacBio SMRT Link software.

DNA sequence analysis were performed at the Scientific Compute Cluster Euler, at ETH, Zurich. The MiSeq data were processed similar to the workflow described in Hartman et al. (2017). Briefly, to improve merging, read ends were trimmed by run if needed (seqtk v.1.2-r94, https://github.com/lh3/seqtk) and subsequently merged (FLASH v.1.2.11) (Magoč and Salzberg 2011) into amplicons. In a next step, CUTADAPT v1.4.2 (Martin 2011) was used to trim off barcode and primer sequence and demultiplex amplicons based on barcode information. Demultiplexed reads were subsequently quality filtered using prinseq-lite v0.20.4 (Schmieder and Edwards 2011). The quality filtered sequences were clustered into operational taxonomic units (OTUs, $\geq 97 \%$ sequence similarity) using usearch v10.0.240 (Edgar 2013). SINTAX (http://biorxiv.org/lookup/doi/10.1101/074161) was used for taxonomic assignments using either SILVA 16S v128 (Quast et al. 2013) for the bacterial community or UNITE v7.2 (Abarenkov et al. 2010) database for the fungal community.

The SMRT sequencing data were processed following Schlaeppi et al. (2016). In brief, $\geq 5$-pass 'reads of insert' (ROI; also, circular consensus sequences CCS) were extracted from the raw data 8) using default parameters. The software mothur v.1.34.4 (Schloss et al. 2009) and flexbar (Dodt et al. 2012) were employed for quality filtering and demultiplexing, respectively. Some raw reads were affected by multiprimer artifacts (Tedersoo et al. 2018) and we employed usearch (v10.0.240) (Edgar 2013) to detect and discarded these reads containing primer sequences within the read. OTU clustering and taxonomic annotation were conducted using the same tools as for the MiSeq data.

Bioinformatics scripts and report files are available as Supplementary Data File S2. The raw sequencing data of the two MiSeq runs and the SMRT sequencing are available from the European Nucleotide Archive under the study accession PRJEB27162.

Statistical analyses. Statistical analyses were performed using R v3.3.2 (R Core Team 2016) within Rstudio (RStudio Team 2015). The effects of $\mathrm{P}$ availability on dry weight, $\mathrm{P}$ content and $\mathrm{K}$ content were assessed with a linear model. Dry weight data were logtransformed to satisfy the assumptions of the linear model (normality of residuals and homoscedasticity). To test for the effect of $\mathrm{P}$ availability on AMF colonization, a generalized linear model was fitted with quasibinomial distribution to account for overdispersion. Rarefaction curves were prepared with the function 'rarecurve' from vegan (Oksanen et al. 2018). For alpha diversity, the data were rarefied to 15,000 sequences 500 times. For each subsample, several diversity indices were estimated: richness $(\mathrm{S})$ is the number of OTUs, $\mathrm{H}$ is the Shannon index from which $\mathrm{D}=\exp (\mathrm{H})$ was calculated (Jost 2007), and Sheldon evenness is $\mathrm{E}=\exp (\mathrm{H}) / \mathrm{S}$ (Sheldon 1969). Analysis of variance (ANOVA) was used to assess the effect of $\mathrm{P}$ application and plant species on the mean of the 500 subsamples for each sample. For the rest of the analysis, the data were filtered (at least four sequences per sample in four samples) to remove low abundant OTUs. The effects of $\mathrm{P}$ availability and plant species on community composition were assessed with permutational multivariate analysis of variance (PERMANOVA) of BrayCurtis dissimilarities and visualized with principal coordinate analysis (PCoA) using vegan and phyloseq (McMurdie and Holmes 2013). The effect of $P$ levels on abundance of each OTU was investigated with edgeR (Robinson et al. 2009) on TMM-normalized data (Robinson and Oshlack 2010) and visualized with ternary plots. TMM-normalized data were used to calculate Spearman rank correlations between OTUs for co-occurrence networks. Positive $(\rho>0.7)$ and significant relationships $(P<0.001)$ were visualized with igraph (Csardi and Nepusz 2006).

Scripts, functions, and support files are available as Supplementary Data File S3. Supplementary Figure S1 visualizes the workflow of the analysis steps.

Identification of fungal endobacteria OTUs by phylogenetic placement. To identify fungal endobacteria OTUs, we preselected candidates in the microbiota dataset using two approaches and then validated their representative sequences by fine mapping to a reference tree of known endobacteria sequences. For the latter, we created a custom database with curated endobacteria 16S rDNA references containing published sequences of Burkholderia-related endobacteria (BRE), such as 'Candidatus Glomeribacter gigasporarum' and Mycoavidus cysteinexigens, and Mycoplasmarelated endobacteria (MRE) retrieved from Glomeromycotina, such as 'Candidatus Moeniiplasma glomeromycotorum', Endogonaceae (Mucoromycotina), and Mortierellomycotina. We pursued two alternative strategies to identify candidate endobacteria OTUs. The first approach was based on sequence clustering and secondly, we employed co-occurrence characteristics from network analysis (e.g., high degree of co-occurrence between fungal and bacterial OTUs) and we explored if this type of information would be useful to identify candidate fungal endobacteria OTUs. 
For the first strategy, we employed usearch (v8) to map the curated endobacteria sequences to the representative bacteria OTUs (bOTU) sequences of the microbiota dataset. We allowed up to $10 \%$ sequence divergence to account for the high variability among $16 \mathrm{~S}$ rDNA endobacteria sequences, in particular from MRE, known to display high level of sequence diversity (Desirò et al. 2018). This approach yielded 22 candidate fungal endobacteria OTUs, which we then placed into the reference tree. For the second strategy, we searched the co-occurrence characteristics from network analysis as follows: we first identified all fungal OTUs (fOTUs) assigned to Glomeromycotina and Mortierellomycotina (we did not identify fOTUs assigned to Endogonaceae; may be linked to the use of universal ITS primers, which tend not to capture this family [Tedersoo et al. 2016]). Then we selected all bOTUs that significantly co-occur with them (Spearman's rho $>0.7$ and $P<0.001$ ). This approach yielded 129 candidate fungal endobacteria OTUs, which we then placed into the reference tree.

Placement to a common phylogenetic tree was achieved by aligning candidate fungal endobacteria OTUs (from clustering or network approaches) to a fungal endobacteria reference dataset. Sequences were aligned with MAFFT (Katoh and Standley 2013). Phylogenetic analyses were carried out with MrBayes v.3.2.6 (Ronquist et al. 2012) and RAxML v.8.2.4 (Stamatakis 2014). Prior to Bayesian phylogenetic reconstructions, best-fit nucleotide substitution models were estimated with jModelTest v.2.1.9 (Darriba et al. 2012). Bayesian analyses were performed running the Markov chain Monte Carlo for 10 million generations under the $\operatorname{TrN}+\mathrm{I}+\mathrm{G}$ nucleotide substitution model. Maximum likelihood analyses were conducted with the automatic 'bootstrapping' option under the GTRCAT nucleotide substitution model. For tree inference, Betaproteobacteria and Mollicutes reference sequences were
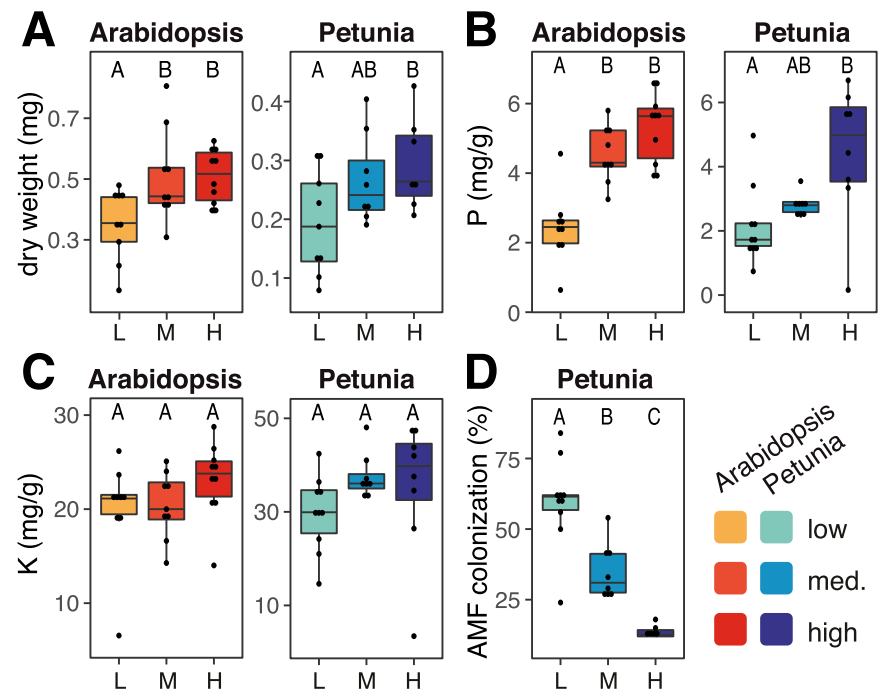

Fig. 1. Effectiveness of manipulated phosphate levels on plant growth, leaf nutrient concentrations and levels of arbuscular mycorrhizal fungi (AMF) root colonization. Arabidopsis (reddish colors, see legend) and Petunia (blueish colors) were grown at low (L), medium (M), and high $(\mathrm{H}$, increasing hue) levels of $\mathrm{P}$ supply and basic plant growth parameters were recorded to confirm the experimental conditions. The first experiment was analyzed for parameters including A, above-ground plant biomass, B, leaf phosphorus levels, C, leaf potassium levels, and D, Petunia root colonization by AMF. A linear model was used to test for effects of $\mathrm{P}$ availability for panels A, (log-transformed data), B, and C, and a quasibinomial generalized linear model for panel $D$. Different letters indicate significant pairwise differences among sample groups $(P<0.05$, Tukey HSD). included together with outgroup sequences belonging to Cyanobacteria. Candidate endobacteria OTUs were considered as BRE or MRE OTUs if they clustered within an endobacteria clade in the reference phylogenetic tree.

Command line and analysis code in $\mathrm{R}$ (including markdown report) as well as the database with curated fungal endobacteria $16 \mathrm{~S}$ rDNA reference sequences are available as Supplementary Data File S4.

\section{RESULTS}

Experimental setup for manipulating phosphate levels. We manipulated the phosphate levels in fertilizer solutions and investigated the responses of the root-associated microbiota of Petunia to varying levels of $\mathrm{P}$ supply. Petunia forms symbiosis with AMF and as a control, we used the nonmycorrhizal model species Arabidopsis. Plants were sown in an experimental soil that is low in plant available $\mathrm{P}$, according to the recommendations for Swiss farmers (Richner and Sinaj 2017). We fertilized the plants with low-, medium-, and high-P containing nutrient solutions. The three specific concentrations of $\mathrm{KH}_{2} \mathrm{PO}_{4}$ were chosen based on Breuillin et al. (2010) to yield three different levels of AMF colonization. In a first experiment, we established our experimental soil system by testing plant growth and AMF colonization in response to the applied phosphate fertilizer levels. We validated that the increasing phosphate concentrations positively affected growth of Arabidopsis and Petunia (Fig. 1A). Leaf nutrient analysis revealed for both species a significant increase of shoot $P$ levels (greater than twofold enhancement from low to high fertilizer treatments, Fig. 1B) while the different fertilization levels affected leaf $\mathrm{K}$ concentrations only marginally (shoot $\mathrm{K}$ tends to increase with fertilization but was not significantly different, Fig. 1C). Hence, phosphate was more limited in our experimental system for plant growth than potassium, which is consistent with the varying amounts of $\mathrm{P}$ (strong gradient) and $\mathrm{K}$ (weak gradient) in the fertilizer treatments (see methods). Microscopic analysis of Petunia roots revealed that the applied fertilizer treatments permitted to establish a gradient ranging from $71.2 \%$ (low-P), over $41.0 \%$ (medium-P) to $4.3 \%$ (high-P) of AMF root colonization (Fig. 1D). In summary, the simple approach of using $\mathrm{KH}_{2} \mathrm{PO}_{4}$ solutions permitted to establish a fertilization gradient from limiting to sufficient $\mathrm{P}$ levels without causing apparent $\mathrm{K}$ limiting conditions.

Evaluation of ITS profiling approaches. We performed a second experiment growing Petunia in the established low-P conditions with the goal to select an ITS PCR approach that permits a reliable quantification of Glomeromycotina fungi while avoiding co-amplification of plant ITS sequences. We evaluated the following PCR approaches to profile root fungal communities: ITS1F and ITS2 (McGuire et al. 2013), fITS7 and ITS4 (Ihrmark et al. 2012), and ITS1F with the reverse complement of fITS7. Community profiles were inspected for the proportions of plant and AMF sequences as well as for fungal diversity. We selected ITS1F and ITS2 because this PCR approach captured low levels of plant sequences at good coverage of AMF and highest levels of taxa richness (Supplementary Fig. S2).

Profiling soil and root microbial communities. To investigate the responses of the root-associated microbiota to varying levels of $\mathrm{P}$ supply, we conducted a third experiment using the experimental conditions and fertilizer solutions with varying $\mathrm{P}$ levels as validated in the first experiment (Fig. 1). We confirmed for the third experiment that the increasing $P$ supply improved plant growth while negatively affected root colonization by AMF (Supplementary Fig. S3). We characterized the soil and root-associated bacterial and fungal communities by sequencing amplicons of the $16 \mathrm{~S}$ rRNA 
gene and the ITS region 1, respectively. With focus on the AMF community and their interacting partners, we sampled tightly root-associated microbes and not the rhizosphere. We obtained $2,196,310$ high-quality bacteria sequences with a median of 36,718 sequences per sample and 3,809,350 high-quality fungal sequences with a median of 54,337 sequences per sample. Bacterial and fungal sequences clustered into 3,701 bacterial operational taxonomic units (bOTUs) and 1,688 fungal OTUs (fOTUs), respectively.

Soil bacteria comprised abundant Acidobacteria, Actinobacteria, Firmicutes, Deltaproteobacteria, and Verrucomicrobia, whereas plant roots were mainly colonized by Betaproteobacteria, Gammaproteobacteria, and Bacteroidetes (Supplementary Fig. S4). The bacteria community composition at phylum rank was not markedly different between Arabidopsis and Petunia.

For fungi, Ascomycota and Basidiomycota were abundant in soil and Petunia root samples. Mortierellomycotina were particularly abundant in soil fungal communities, whereas a high number of Glomeromycotina was found in Petunia roots, which varied as a function of the applied phosphate levels. Consistent with the levels of AMF root colonization measured by microscopy, Glomeromycotina were most abundant in Petunia roots under low-P conditions and decreased in proportion with increasing $\mathrm{P}$ availability (Fig. 2A). The cumulative relative abundance of Glomeromycotina sequences was significantly positively correlated (adj. $R^{2}=0.59 ; P<0.001$ ) with the rate of AMF root colonization as assessed by microscopy (Fig. 2B).

We noted that the root fungal community of Arabidopsis was dominated by sequences belonging to Olpidiomycotina. Most of these sequences belonged to fOTU1 (assigned to Olpidium brassicae), which accounted up to $94.6 \%$ of the sequences in Arabidopsis but only $0.8 \%$ of the sequences in Petunia samples and $1.7 \%$ of the sequences in unplanted soil samples. To exclude that this is a technical peculiarity of MiSeq, we confirmed the dominance of $O$. brassicae by sequencing the entire ITS region (PCR primers ITS1F and ITS4) using SMRT sequencing. We refer to the Supplementary Data File S5 for a more detailed comparison of the sequencing approaches. In brief, the SMRT-sequencing based community profiles also avoided amplifying plant sequences while abundantly capturing the AMF. Both methods have their inherent technical advantages with the MiSeq approach offering enhanced throughput and sampling depth, while the SMRT-sequencing method provides enhanced taxonomic resolution. Albeit a few
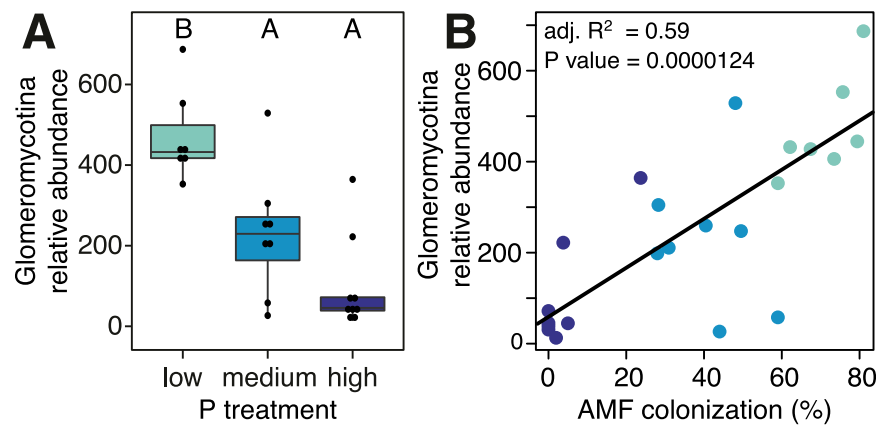

Fig. 2. Abundance of arbuscular mycorrhizal fungi (AMF) in Petunia roots. A, Quantification of AMF based on the relative abundance of Glomeromycotina sequences in the microbiota profiles. Relative abundances were calculated from total sum normalized data. A quasibinomial generalized linear model was used to test for effects due to $P$ availability. Different letters indicate significant pairwise differences between sample groups $(P<0.05$, Tukey HSD). This data comes from experiment three and was correlated $\mathbf{B}$, with the levels of AMF root colonization as measured in the same samples by microscopy. quantitative differences, the two approaches reproduce overall similar taxonomic compositions and revealed remarkably similar biological patterns.

Phosphate induced variation in microbial diversity. In the following, we used the MiSeq-based fungi profiles as they were obtained using the same sequencing platform as the bacteria and because of the enhanced sequencing depth. Microbial richness was highest in unplanted soil, followed by Petunia and then Arabidopsis roots (Supplementary Fig. S5). ANOVA confirmed the differences in alpha diversity between the two plant species and further uncovered an effect by the different $\mathrm{P}$ levels on the bacteria community (Supplementary Fig. S6, Supplementary Table S1). Bacterial richness, diversity, and evenness were generally higher in Petunia compared with Arabidopsis and generally increased with increasing $\mathrm{P}$ concentrations. With the dominance of $O$. brassicae, fungal richness, diversity, and evenness were markedly lower in Arabidopsis compared with Petunia.

Utilizing principal coordinate analysis (PCoA) of Bray-Curtis dissimilarities, we found compositional differences in microbial communities due to the tested experimental factors sample type, $\mathrm{P}$ fertilizer and between the two plant species. Consistent with previous work (Bulgarelli et al. 2012; Hartman et al. 2018), bacteria and fungi differed markedly between the sample types of unplanted soil and roots (Supplementary Fig. S7). Comparing the root communities of the two plant species, fungal communities were more divergent between Arabidopsis and Petunia roots compared with bacteria communities (Fig. 3). PERMANOVA, finding significant plant species effects on both bacterial and fungal communities (Supplementary Table S2), confirmed that the two species varied more in their fungi ( $76 \%$ of variation) compared with their bacteria communities $(16 \%)$.

The effects of varying $\mathrm{P}$ levels were apparent in bacterial communities of both Arabidopsis and Petunia by clustering along axis 1 following the gradient in phosphate levels (Fig. 3A), whereas for fungi, a clustering by $\mathrm{P}$ levels was only manifested in Petunia (Fig. 3B). PERMANOVA confirmed over both species a significant $\mathrm{P}$ fertilizer effect for the bacteria as well as for the fungi albeit weaker. Consistent with Figure 3B, a significant interaction term was found for the fungi revealing that the communities differ in their response to the $\mathrm{P}$ treatments between the two species. To approximate the effect sizes of $\mathrm{P}$ treatments on the Petunia and Arabidopsis root microbial communities, we inspected the $R^{2}$
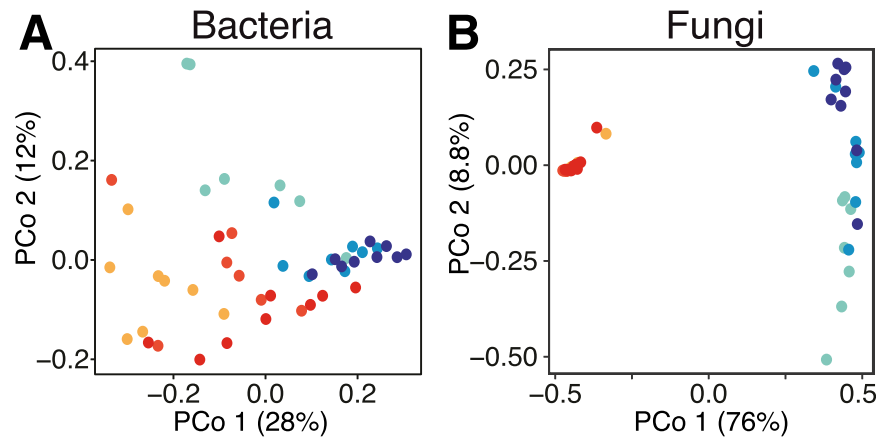

Fig. 3. Effects of plant species and P-levels on community composition. Unconstrained ordinations with PCoA using Bray-Curtis dissimilarities were performed on the $\mathbf{A}$, bacterial and $\mathbf{B}$, fungal communities associated with roots. Samples were colored following the color scheme defined in Figure 1 (Arabidopsis and Petunia with reddish and blueish colors, respectively, and the increasing $\mathrm{P}$ availability (low, medium, and high) are marked with increasing hue. 
values of PERMANOVA applied to the data of each plant separately. While P levels explained 25.3 and $28.1 \%$ of significant variation in Petunia root bacterial and fungal communities, respectively, it accounted for 18.5 and $10.0 \%$ of variation in the Arabidopsis root microbial communities (Supplementary Table
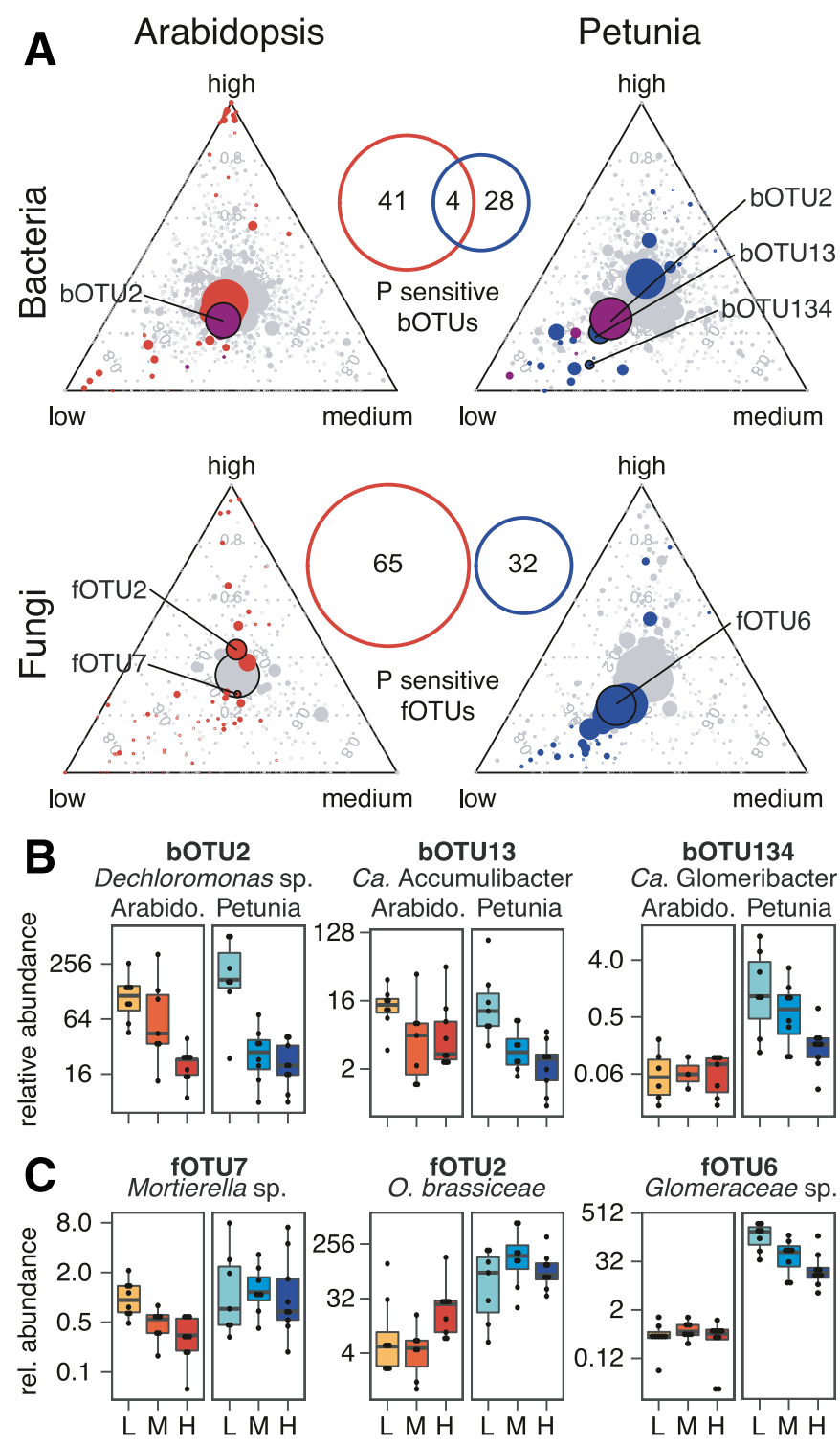

Fig. 4. Identifying operational taxonomic units (OTUs) that vary in abundance in response to phosphate supplementation. OTUs that were differentially abundant between low- $P$ and high-P conditions were separately identified for the bacteria and fungi, both in Arabidopsis and Petunia (edgeR analysis, high-P versus low-P, FDR $<0.05$ ). A, The ternary plots depict individual OTUs (in circles), sized by their relative abundance, and the position of the OTUs in the triangle reflects their proportional abundance in low-, medium-, and high-P samples. $\mathrm{P}$ responsive OTUs are colored in red (Arabidopsis), blue (Petunia), or purple (found in both species) and nonaffected OTUs are colored in gray. The dominant Olpidium brassicae fOTU1 in the Arabidopsis fungal community is gray and marked with a black border. The number of $P$ responsive OTUs and their overlap between species is given with the Venn diagrams. B and C, The relative abundances (per mil, \%o) of a few representative $P$ responsive bacterial and fungal OTUs in low $(L)$, medium $(\mathrm{M})$, and high $(\mathrm{H}) \mathrm{P}$ conditions, which are also indicated in the ternary plots. Of note: fOTU2 (average abundance of $21 \%$ in Arabidopsis roots) is a different $O$. brassicae OTU compared with dominant fOTU1 (average abundance of $946 \%$ ).
S3). Without a significant effect on Arabidopsis fungi, this indicates that varying $\mathrm{P}$ supplementation can account on average for $24 \%$ of variation in plant root microbiota composition.

Identifying microbes with P-dependent abundances. Next, we identified OTUs being differentially abundant between low and high-P conditions using edgeR (Robinson et al. 2009). In total we found $2.2 \%$ bOTUs and $6.3 \%$ fOTUs varying in abundance due to the differing $\mathrm{P}$ levels in Petunia, while $3.1 \%$ bOTUs and $13 \%$ fOTUs were differentially abundant in Arabidopsis (Fig. 4A). With the exception of four bOTUs, distinctive sets of differentially abundant bOTUs and fOTUs were found for Arabidopsis and Petunia, suggesting that the two plant species have contrasting microbial responses to low-P conditions. Among the four shared bOTUs was a prominent Dechloromonas sp. (bOTU2), which is more abundant under low-P conditions in both plant species (Fig. 4B). Bacteria from different taxonomic lineages were abundant under low or high-P conditions (Supplementary Table S4). The most abundant Arabidopsis root bacteria included Burkholderiales, Bdellovibrionales, and Rhodocyclales under low-P conditions, whereas taxa from the Chthoniobacterales, Planctomycetales, and Verrucomicrobiales were enriched under high-P conditions. Under low-P conditions, the abundant Petunia root bacteria included also members of the Burkholderiales and Rhodocyclales, whereas under high-P conditions, a slightly different set of bacteria, including a Flavobacterium sp. (Flavobacteriales), a Tahibacter sp. (Xanthomonadales) and again members of the Verrucomicrobiales were abundant. Examples of highly abundant and low-P specific Burkholderiales and Rhodocyclales members include a Dechloromonas sp. (bOTU2) and a Candidatus Accumulibacter (bOTU13, Fig. 4B). Among the Petunia root bacteria, which are enriched under low-P conditions, we noticed a bOTU assigned to 'Candidatus Glomeribacter gigasporarum' (bOTU134, Fig. 4B), which presents an endobacterium associated with lineages in the AMF family Gigasporaceae (Bianciotto et al. 2003).

Similar to bacteria, different fungal lineages varied in abundance to low or high-P conditions in Arabidopsis and Petunia. In Arabidopsis, besides many low abundant and often taxonomically poorly resolved fungi, the distinct group of Mortierellomycotina (e.g., fOTU7, Fig. 4C) was enriched under low-P conditions. Under high-P conditions, the abundant fungi $O$. brassiceae (Olpidiales, fOTU2 [ $\neq$ the dominant fOTU1], Fig. 4C), Hygrophoraceae sp. (Agaricales, fOTU10), and Cadophora sp. (Helotiales, fOTU14) were found besides numerous low abundant fOTUs. While in Petunia only a handful of diverse and low abundant fungi were enriched under high-P conditions, we found a large group of 28 mycorrhizal fOTUs enriched in the low-P treatment. These mycorrhizal fOTUs belonged mostly to the order Glomerales and included numerous abundant members such as Funneliformis and Glomus spp. (e.g., fOTU6 in Fig. 4C).

Phosphate-induced changes in microbial co-occurrence patterns. Finally, we utilized co-occurrence network analysis to find pairs or groups of microbes with a similar abundance behavior along the gradient of $\mathrm{P}$ fertilizer. Co-abundance presents a prerequisite for cooperation among microbes and we speculated to identify possible candidate cooperation partners that may contribute to support plant growth under low-P conditions. Figure 5A visualizes the significant positive pairwise correlations between root microbiota members (bOTU-bOTU, fOTU-fOTU, and bOTUfOTU) of Petunia and Arabidopsis growing in conditions with low, medium or high-P application. We then partitioned the network into discrete community modules and mapped the bOTUs and fOTUs with P-dependent abundances into the network and modules. While we did not find groups of co-occurring OTUs (= modules) that were enriched in high-P conditions, we found two 
major modules, 'M1' and 'M26', that comprised high proportions of OTUs (Fig. 5B) being specifically abundant under low-P conditions (Fig. 5C). The module 'M1' comprised only bacteria, mainly belonging to the Betaproteobacteria orders Burkholderiales and Rhodocyclales (Supplementary Table S5). In contrast, the module 'M26' grouped a set of five taxonomically diverse bacteria lineages with a large set of fOTUs primarily belonging to the order Glomerales. These fOTUs represented almost all AMF fOTUs in the dataset and interestingly, they co-occurred with the Candidatus Glomeribacter bOTU134.

The same analysis was conducted for Arabidopsis and revealed a module 'M27' with co-occurring bacteria and fungi OTUs that were specifically abundant under high-P conditions (Supplementary Fig. S8). This module grouped diverse bacteria members including Planctomycetes and Verrucomicrobia and a diverse set of fungi. The module 'M19' held low abundant and taxonomically diverse bacteria and fungi that favored intermediate P levels. The module 'M4' comprised abundantly co-occurring bacteria and fungi under low-P conditions, belonging mainly to diverse Proteobacteria and Ascomycota or unknown fungi, respectively. With the exception of a few bacteria, the modules with low-P enriched OTUs of Arabidopsis and Petunia had specific compositions (Fig. 5D), which is consistent with the species-specific root microbiota changes to low-P condition.

Fungal endobacteria. To understand whether bacterial communities associated with the Arabidopsis or Petunia root microbiota could include fungal endobacteria, we aligned candidate bOTU sequences to a database of curated fungal endobacteria sequences (see methods). Phylogenetic placement confirmed bOTU134 as a
Candidatus Glomeribacter. The closest match in the reference fungal endobacteria database was a sequence obtained from an endobacterium of the AMF species Scutellospora pellucida of the family Gigasporaceae (Fig. 6A, Supplementary Fig. S9 and 10). bOTU134 is co-occurring most closely with the AMF OTUs fOTU103 $(\rho=0.71, P<0.001)$ and fOTU165 $(\rho=0.73, P<0.001$, Fig. 6A inset), both classified as members of the Glomeraceae family. Phylogenetic placement analysis revealed additional five candidate OTUs that map to Burkholderia- and four Mycoplasmarelated endobacteria (Fig. 6). However, either we did not find cooccurrence evidence with fungal OTUs (bOTUs 330 and 778) or they were only detected with a handful of reads in a few samples and we did not include them for network analysis. Interestingly, bOTUs 330 and 778 were, similar to bOTU134, significantly enriched in abundance under low-P conditions. The use of microbiota network characteristics alone was generally not indicative for identifying fungal endobacteria OTUs, by contrast the clustering-based approach proved to function well. In summary, the combined sequencing of bacteria and fungi and co-occurrence analysis revealed an OTU, closely mapping to an endobacterium of AMF, that had a consistent abundance behavior with mycorrhizal OTUs along the $\mathrm{P}$ gradient.

\section{DISCUSSION}

In this study, we examined fertilizer induced changes of the root microbiota investigating mycorrhizal Petunia plants and the nonmycorrhizal model plant Arabidopsis. With varying $\mathrm{KH}_{2} \mathrm{PO}_{4}$ in the fertilizer solutions, we manipulated both the fertilizer's $\mathrm{P}$ and $\mathrm{K}$
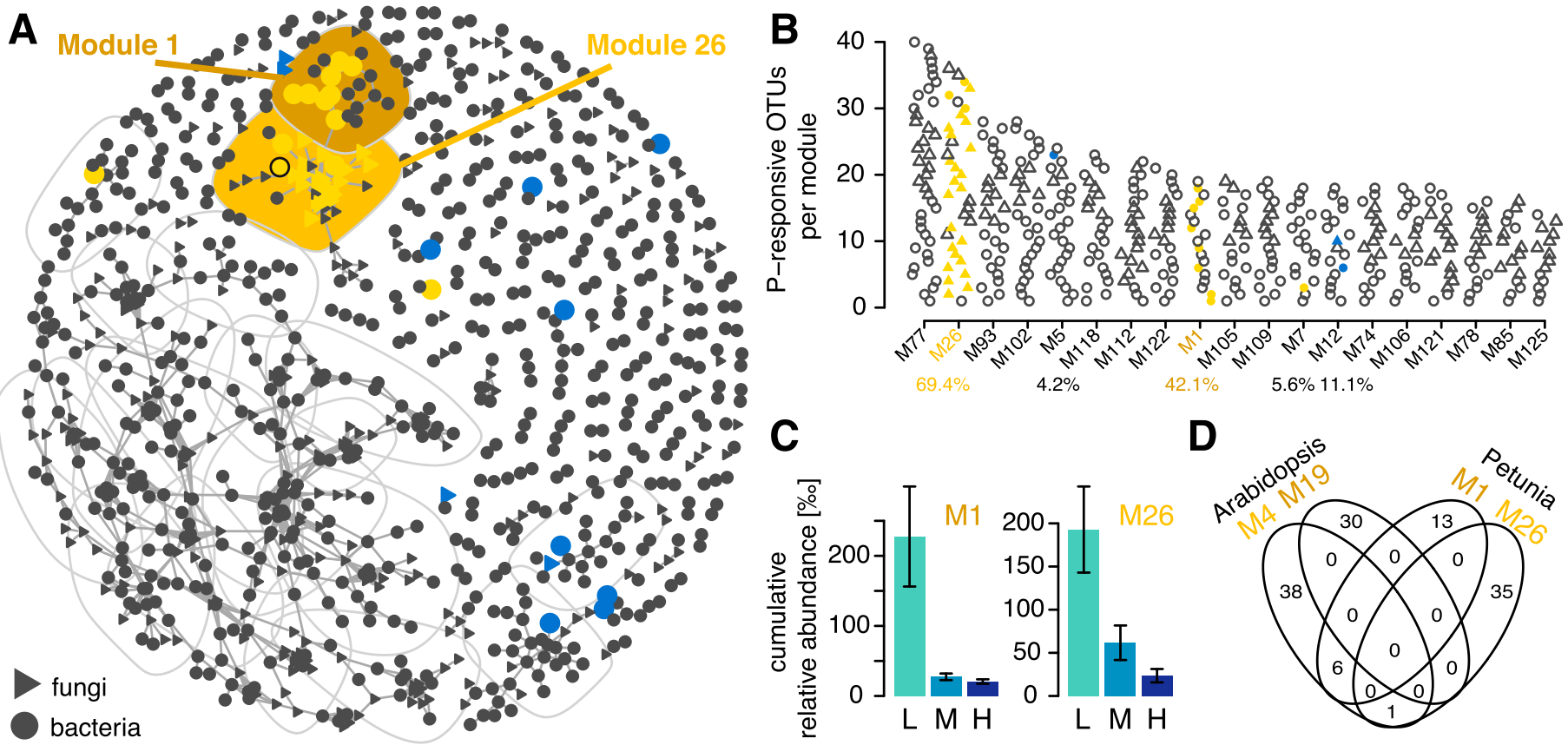

Fig. 5. Petunia root microbiota co-occurrence patterns along varying levels of $P$ supply. A, Co-occurrence networks visualize the significant positive pairwise correlations $(\rho>0.7, P<0.001$; indicated by links between operational taxonomic units [OTUs]) between bacteria (circles) and fungi (triangles) OTUs in Petunia root communities. P-dependent OTUs, which are abundant under low- and high-P conditions, are colored in yellow and blue, respectively. The 20 network modules comprising highest numbers of OTUs are rimmed with gray lines with the modules containing high proportions of P-responsive OTUs being shaded in yellow and blue. Candidatus Glomeribacter bOTU134 in module 'M26' is marked in yellow with a black border. B, Top 20 most populated modules, ranked by decreasing numbers of OTUs (bOTUs in circles; fOTUs as triangles) with low- and high-P responding OTUs being colored in yellow and blue, respectively. Percentages below the $\mathrm{x}$-axis report the proportion of $\mathrm{P}$ dependent OTUs present in each module. C, Cumulative relative abundance (as per mil, \%) of all bacteria and fungi OTUs in the $P$ responsive modules in low (L), medium (M), and high (H) $P$ conditions. The cumulative relative abundance indicates the overall response of the microbes in the $\mathbf{P}$ responsive modules. $\mathbf{D}$, Number and overlap of OTUs in the low-P responsive modules of Arabidopsis and Petunia are shown with the Venn diagram. 

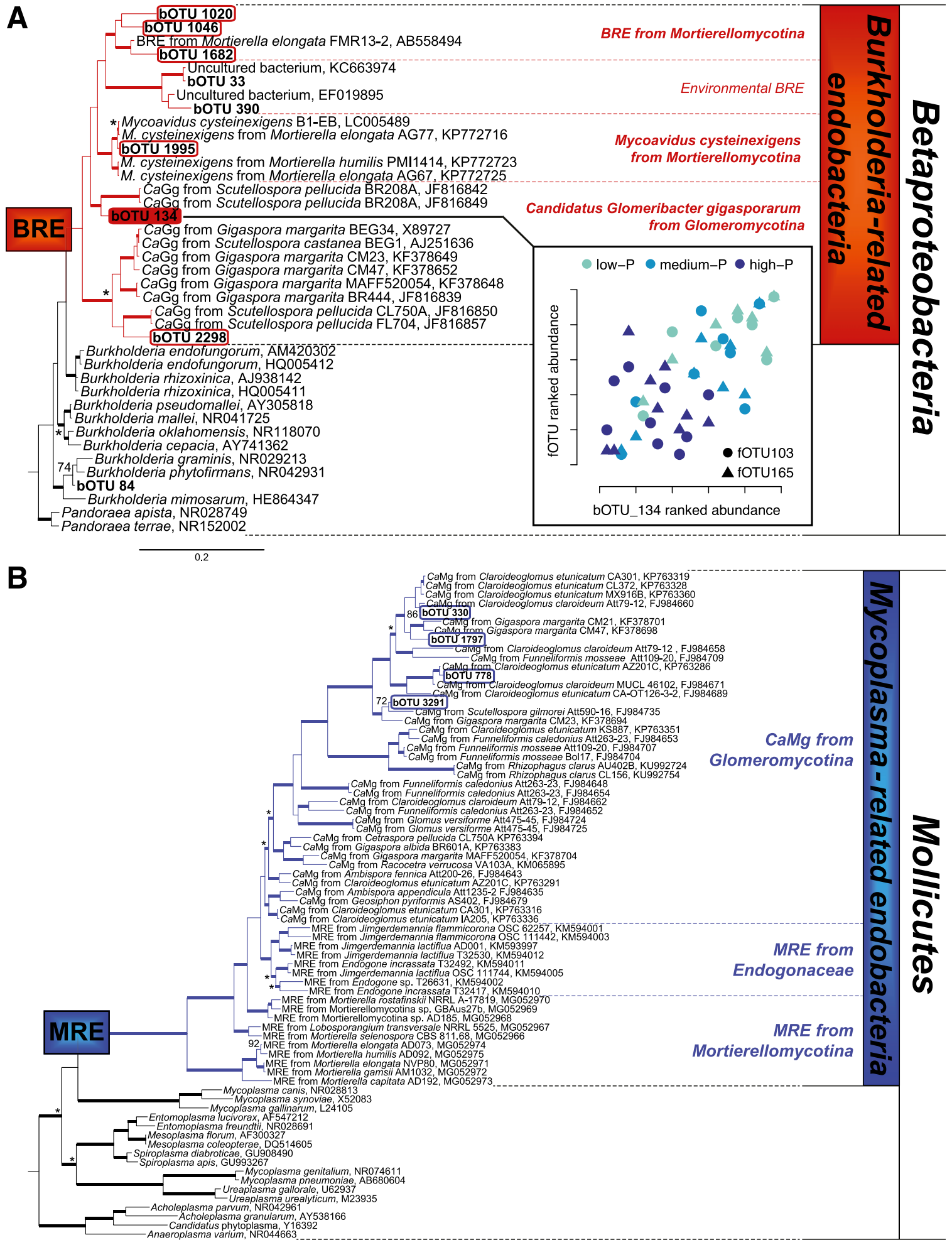

Fig. 6. Phylogenetic placement of fungal endobacteria operational taxonomic units (OTUs) from candidates identified by the clustering approach. Simplified trees summarizing the Burkholderia-related endobacteria (BRE, a) and Mollicutes-related endobacteria (MRE, b). A, Four candidate BRE OTUs cluster within two clades encompassing BRE sequences from Mortierellomycotina fungi. In detail, bOTU 1995 is sister to the type strain of Mycoavidus cysteinexigens, whereas bOTUs 1020, 1046, and 1682 cluster with an undescribed BRE hosted in Mortierella elongata. Two bOTUs (134 and 2298 ) cluster within two clades encompassing 'Candidatus Glomeribacter gigasporarum' (CaGg) sequences retrieved from Scutellospora pellucida (Glomeromycotina). Two bOTUs (33 and 390) cluster within a new BRE clade, together with putative environmental BRE sequences. B, Four candidate MRE OTUs cluster within different clades encompassing 'Candidatus Moeniiplasma glomeromycotorum' (CaMg) sequences from Glomeromycotina fungi. In detail, bOTUs 330 and 778 cluster with CaMg hosted in several strains of Claroideoglomus spp., whereas bOTUs 1797 and 3291 cluster with CaMg hosted in several strains of Gigaspora margarita and Scutellospora pellucida. The trees show the topology obtained with the Bayesian method. Branches with Bayesian posterior probabilities $(\mathrm{BPP}) \geq 0.95$ and maximum likelihood (ML) bootstrap support values $\geq 70$ are thickened; asterisks $\left(^{*}\right)$ indicate branches with BPP $\geq 0.95$ but ML bootstrap support values $<70$; ML bootstrap support values $\geq 70$ are shown for branches having BPP $<0.95$. Sequences generated in this study are in bold. 
levels resulting in low-P conditions being also partially limited for $\mathrm{K}$. However, because the fertilizer solutions contained basic levels of $\mathrm{K}$ (see methods), we rate the influence of $\mathrm{K}$ lower compared with $\mathrm{P}$. Measuring leaf mineral levels confirmed that $\mathrm{P}$ was more limited than $\mathrm{K}$ in our experimental system as plants had significantly lower $\mathrm{P}$ levels in low-fertilizer conditions, while leaf $\mathrm{K}$ concentrations were not significantly affected (Fig. 1). To simplify matters, we refer to the main factor of varying the phosphate levels, but we do not exclude that the observed microbiota changes may also be due to the concomitant partial shortage of potassium in low-P and medium-P treatments.

We find that the composition of the root microbiota changes in response to varying $\mathrm{P}$ fertilization levels and we find different root microbiota compositions under low-P conditions in the two plant species. Under low-P conditions, we confirmed a substantial colonization by AMF in Petunia together with numerous bacteria of the Burkholderiales and Rhodocyclales, whereas Arabidopsis roots hosted mainly Mortierellomycotina fungi and abundant bacteria from the Burkholderiales, Bdellovibrionales, and Rhodocyclales. However, the root microbiota of the two plants contained different members (at the OTU level) of these taxonomic lineages. A next step to deepen our understanding is to compare the functional repertoire of the Arabidopsis and Petunia root-associated microbes that are enriched under low-P conditions. For soil microbial communities, Yao et al. (2018) found an adaptive allocation of specific genes in response to low $\mathrm{P}$ availability using a metaproteogenomic approach. Low-P conditions enhanced abundances of 3-phytase, phosphodiesterase, phospholipase and exoribonuclease genes revealing that soil microbial communities enhanced their genetic capabilities to recycle and extract $\mathrm{P}$ from recalcitrant minerals and organic compounds. A potential adaptive enrichment of $\mathrm{P}$ cycling genes for the plant-associated microbiota raises additional questions such as to what extend they contribute to plant growth under the nutrient constraining conditions or what type of genetic capabilities become enhanced under low-P conditions on roots of mycorrhizal and nonmycorrhizal plants?

Fungal endobacteria. The aforementioned groups of $\mathrm{P}$ dependent and differentially abundant microbes responded simultaneously to the different levels of $\mathrm{P}$ application. Among these co-occurring microbes, we found sequences of fungal endobacteria and sequences of their potential hosts, presenting a well-known example of highly specific multitrophic microbial interactions. To our knowledge, this is the first report of "fungal endobacteria OTUs" of Mucoromycotina in a community sequencing-based plant microbiota study. Above all, the experimental design with low- to high-P conditions was instrumental to identify sequences from fungal endobacteria in the dataset. A first hint for the presence of fungal endobacteria sequences came from the statistical approach to find differentially abundant bOTUs between low and high-P conditions, revealing the enrichment of sequences under low-P conditions that mapped to a Candidatus Glomeribacter (Fig. 4B). Of note, bOTU134 was almost overlooked, as a reliable taxonomy assignment (confidence $>0.7$ ) was only available down to family level, while deeper data exploration indicated a link with endobacteria (genus-level assignment was Candidatus Glomeribacter, confidence 0.34 ). The second hint that pointed to potential fungal endobacteria and their hosts were the co-abundance patterns, which grouped bOTUs including Candidatus Glomeribacter bOTU134 with numerous Glomeromycotina fOTUs. Here again, experimental design was instrumental because the co-occurrence analysis groups microbe sequences with similar abundances in samples from low- to high-P conditions. Finally, we relied on phylogenetic placement to confirm that the bOTU134 sequence corresponded to a sequence of Candidatus Glomeribacter, which is derived from a previously isolated exemplar from the AMF species Scutellospora pellucida
(Fig. 6). The logical next step is now to validate such an AMFendobacteria relationship as proposed by short DNA sequences, e.g., by performing bacterial and fungal sequence analyses from single fungal spores collected from the experimental soil to confirm whether the locally occurring AMF species indeed harbor the identified endobacterial OTUs.

The identification of bOTU134 as a potential fungal endobacterium prompted us to search for additional potential fungal endobacteria in the Arabidopsis or Petunia root microbiota. We compared two strategies, co-occurrence characteristics and sequence similarity, for their usefulness to identify fungal endobacteria from root microbiota data. For the co-occurrence characteristics strategy, we selected all bOTUs that significantly cooccurred with fOTUs from fungal lineages known to host endobacteria, and then placed the resulting 129 candidate bOTUs into the endobacteria reference tree. For the second strategy, we clustered all sequences of the curated endobacteria database with all representative bOTU sequences of the microbiota dataset, and then placed the resulting 22 candidate bOTUs with the highest sequence similarity into the endobacteria reference tree. In addition to bOTU134, two abundant (bOTUs 330 and 778) and seven lowabundant endobacteria OTUs were identified by the two strategies. While both approaches identified candidate endobacteria, the clustering-based approach functioned more efficiently as also low abundant candidates were identified. For bOTUs 330 and 778, we did not find co-occurrence evidence with fungal OTUs, so we did not consider them as robust fungal endobacteria OTUs.

There are probably multiple reasons why fungal endobacteria and their hosts were largely neglected in microbiota studies so far. Reasons include the low taxonomic resolution of short-read community data or the underrepresentation of reference fungal endobacteria sequences in commonly used taxonomy databases. Here, only the combined sequencing of bacteria and fungi together with the dedicated experimental design to manipulate the abundance pattern of fungal hosts and the curated fungal endobacteria database permitted to designate a few OTUs as 'fungal endobacteria OTUs'. Our study demonstrates that microbiota and/or metagenomic datasets represent useful tools to investigate endobacterial-fungal interactions in their true ecological context. Possibly, such cultivation-independent methods can point to further endobacterial-fungal partnerships. Since fungal endobacteria are ecologically relevant for the fitness of their (plant-associated) fungal hosts (Desirò et al. 2018; Salvioli et al. 2016; Uehling et al. 2017), they may also relay some benefits or detriments to the host plant of the fungus (Bonfante and Desirò 2017). Hence, mycorrhizal plants, their colonizing fungi along with their endobacteria form an entity of a multi-kingdom symbiosis. As put forward by the holobiont concept (Vandenkoornhuyse et al. 2015), the importance of multikingdom microbe-microbe interactions for plant performance is not only true for endobacteria and their fungal hosts but also in general between root bacteria and root fungi. For instance, root bacteria are essential to protect plants against pathogenic root fungi (Durán et al. 2018).

Quantifying AMF in plant roots. Although specific sequencing methods to quantify AMF in plant roots (Öpik et al. 2009; Schlaeppi et al. 2016) are available, we sought to assess AMF in plant roots in the context of the whole fungal diversity. In the search for a suitable PCR approach that avoided amplification of plant ITS sequences, we also wanted that AMF would be well captured in plant roots unlike other plant root-fungi profiling methods (Hartman et al. 2018; Ihrmark et al. 2012). The Illumina approach by McGuire et al. (2013), although reporting soil fungal profiles, indicated that PCR primers ITS1F and ITS2 would permit to abundantly capture AMF and this approach turned out to be successful on plant roots, too. 
Prior to community sequencing, we had evaluated the effectiveness of our experimental $\mathrm{P}$ gradient by confirming that AMF abundantly colonize Petunia roots under low-P but not under high-P conditions (Fig. 1C). We performed this quality control using the traditional "magnified intersection" (McGonigle et al. 1990) microscopy method on equivalent subsamples as the ones that were used for the sequencing. The microscopy method relies on staining cleared roots, interpreting and enumerating the different fungal structures following a defined counting scheme under the microscope. Compared with the sequencing-based quantification of AMF in plant roots, the microscopy method appears disadvantageous as it is prone to operator-to-operator variation, is time consuming and lacks throughput, discrimination between AMF species as well as the context of the whole fungal diversity. Nevertheless, we found a reliable agreement between the two methods with a significant positive correlation (Fig. 2B). In summary, the MiSeq-based community profiling approach with the PCR primers ITS1F and ITS2 avoided amplification of plant ITS sequences, abundantly displayed the AMF and independently reproduces AMF colonization patterns of plant roots.

O. brassicae. The root microbiota of Arabidopsis, whether profiled with MiSeq or SMRT-sequencing, comprised abundant sequences assigned to $O$. brassicae, which is a common rootinfecting fungal pathogen of Brassicaceae plants (Lay et al. 2018). When harvesting the roots, the Arabidopsis plants were without signs of disease and we learned after the community sequencing that the fungus was present in the soil and had become abundant in the root tissue. Dominance of $O$. brassicae OTUs was observed in previous studies (Durán et al. 2018; Fabiańska et al. 2018; Lay et al. 2018; Tkacz et al. 2015). Similar to our study, Lay et al. (2018) examining canola, wheat, and pea roots, also found a Brassicaceae-specific enrichment of an $O$. brassicae OTU. Interestingly and consistent with our study (Fig. 4C), Fabiańska et al. (2018) also found elevated levels of $O$. brassicae OTUs under high$\mathrm{P}$ conditions, suggesting that enhanced $\mathrm{P}$ fertilization facilitates root colonization by this fungus. Because different sequencing approaches were used, abundant $O$. brassicae are seen in different Brassicaceae species and different soil types, we consider the dominance of $O$. brassicae in Arabidopsis roots in our study rather a true biological observation than a technical artifact. The highly abundant OTUs of putatively pathogenic Olpidium fungi demand for independent replicate experiments to clarify the Arabidopsis' fungal community responses under low-P especially because of the link between the plant's phosphorus starvation response and immunity (Castrillo et al. 2017). Furthermore, short-read microbiota profiling has probably insufficient resolution to reveal intra-OTU diversity that might explain the apparent lack of pathological symptoms and future work with isolated exemplars is needed to clarify the ecology of this fungus.

P-dependent microbiota abundance patterns. Similar to maize (Gomes et al. 2018), the root microbiota of Petunia was also enriched in Burkholderiaceae bacteria and Glomeromycota fungi in low $\mathrm{P}$ conditions. We found different microbial patterns in response to low-P conditions for Petunia and Arabidopsis with abundant Glomeromycotina and Mortierellomycotina fungi, respectively (Fig. 4). Similar to the functioning of AMF in plant $\mathrm{P}$ provision, there are some reports that Mortierella spp. support P nutrition of plants (Alori et al. 2017). Although represented by different sequence groups, bacteria of Burkholderiales and Rhodocyclales were abundant in both species. Bacteria of both orders, including members that attach to AMF hyphae, are well known for their ability to solubilize and mobilize P (Alori et al. 2017; Sharma et al. 2013; Taktek et al. 2015). Candidatus Accumulibacter being abundant in Petunia under low-P conditions (bOTU13, Fig. 4B) as well as the Dechloromonas sp. (bOTU2, enriched in low-P, both plant species) are both intriguing root bacteria, as they are capable of polyphosphate metabolism, which has been implicated in stress response to low nutrients in the environment (Flowers et al. 2013; Rao and Kornberg 1996). In summary, it appears that nonmycorrhizal Arabidopsis and mycorrhizal Petunia form different microbial associations under low-P conditions.

In contrast to Robbins et al. (2018), we found marked root microbiota changes in Arabidopsis between low-P and high-P fertilized treatments. A plausible reason for stronger $\mathrm{P}$ effects on the root microbiota is that our approach with the sand-soil mixture created stronger $\mathrm{P}$ limiting conditions (1.05 mg of $\mathrm{P} \mathrm{kg}^{-1}$ of soil). For comparison, the low-P control soil of the long-term P fertilization experiment ( $\mathrm{P} 1$ plots did not receive phosphate amendments over the last 65 years) studied by Robbins et al. (2018) contained $2.3 \mathrm{mg}$ of $\mathrm{P} \mathrm{kg}^{-1}$ of soil. Of note, values are directly comparable as they were analyzed in the same professional soil laboratory with the same method. Moreover, we established a steeper gradient between

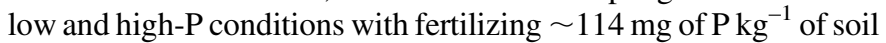
as high-P condition compared with $\sim 12 \mathrm{mg}$ of $\mathrm{P} \mathrm{kg}^{-1}$ in the longterm $\mathrm{P}$ fertilization experiment. The stronger $\mathrm{P}$ limiting conditions in our study were also reflected in the different rosette biomass data in both studies as we find a twofold reduction in median rosette biomass whereas they measured at maximum $1.5 \times$ effect in low-P soils.

In our study we used a low-P adapted soil microbiota as source for the root microbiota under medium- and high-P conditions. An emerging question of this setup is whether the assembly of the root microbiota is not eventually a biased outcome due to a nonadapted soil community? To clarify how adaptation of the soil microbiota is connected to the assembly of the root microbiota, it will be necessary to determine how fast a low-P adapted soil microbiota adapts to the applied fertilizers (Leff et al. 2015) and how strongly the plant's nutritional status dictates the assembly of the root microbiota (Fabiańska et al. 2018). The focus of this study was the root microbiota and the few soil samples, collected as outgroup controls, do not permit a rigorous assessment of fertilizer effects in soil samples and compare them to root samples. Future work based on time-series sampling is needed to clarify the temporal adaptation of established soil and root microbiotas to fertilizer applications and changes in nutrient availability.

Concluding remarks. The root microbiotas of Arabidopsis and Petunia contained different sets of microbes under low-P conditions. Assuming that these microbes are specifically recruited under low-P and that they improve plant growth, as shown for AMF, this would have consequences for agricultural applications. Plantspecies specific microbiota responses suggest that for supporting different plant species in $\mathrm{P}$ nutrition, different $\mathrm{P}$-transporting, $\mathrm{P}$ solubilizing, and/or P-mineralizing microbes are needed. Possibly this explains the high context dependency of successful field applications with microbial products.

\section{ACKNOWLEDGMENTS}

We thank A. Held (Agroscope) for support in amplicon library preparation; N. Savant for help with pot experiments; D. Reinhardt from the University of Fribourg for Petunia seeds; M. Zuber and D. Bürge from Agroscope for the analysis of the leaf nutrient content and soil chemical properties; L. Poveda and A. Patrignani from the Functional Genomics Center in Zürich for technical support in MiSeq- and SMRT-sequencing, respectively; and C. Soneson and M. Robinson from the University of Zürich for input regarding edgeR analysis. 
LITERATURE CITED

Abarenkov, K., Nilsson, R. H., Larsson, K. H., Alexander, I. J., Eberhardt, U., Erland, S., et al. 2010. The UNITE database for molecular identification of fungi-recent updates and future perspectives. New Phytol. 186:281-285.

Almario, J., Jeena, G., Wunder, J., Langen, G., Zuccaro, A., Coupland, G., et al. 2017. Root-associated fungal microbiota of nonmycorrhizal Arabis alpina and its contribution to plant phosphorus nutrition. Proc. Natl. Acad. Sci. USA 114:E9403-E9412.

Alori, E. T., Glick, B. R., and Babalola, O. O. 2017. Microbial phosphorus solubilization and its potential for use in sustainable agriculture. Front Microbiol. 8:971.

Bergkemper, F., Schöler, A., Engel, M., Lang, F., Krüger, J., Schloter, M., et al. 2016. Phosphorus depletion in forest soils shapes bacterial communities towards phosphorus recycling systems. Environ. Microbiol. 18:1988-2000.

Bianciotto, V., Lumini, E., Bonfante, P., and Vandamme, P. 2003. "Candidatus Glomeribacter gigasporarum" gen. nov., sp. nov., an endosymbiont of arbuscular mycorrhizal fungi. Int. J. Syst. Evol. Microbiol. 53:121-124.

Bodenhausen, N., Horton, M. W., and Bergelson, J. 2013. Bacterial communities associated with the leaves and the roots of Arabidopsis thaliana. PLoS One 8: e56329.

Bonfante, P., and Desirò, A. 2017. Who lives in a fungus? The diversity, origins and functions of fungal endobacteria living in Mucoromycota. ISME J. 11: 1727-1735.

Breuillin, F., Schramm, J., Hajirezaei, M., Ahkami, A., Favre, P., Druege, U., et al. 2010. Phosphate systemically inhibits development of arbuscular mycorrhiza in Petunia hybrida and represses genes involved in mycorrhizal functioning. Plant J. 64:1002-1017.

Bulgarelli, D., Rott, M., Schlaeppi, K., Ver Loren van Themaat, E., Ahmadinejad, N., Assenza, F., et al. 2012. Revealing structure and assembly cues for Arabidopsis root-inhabiting bacterial microbiota. Nature 488:91-95.

Busby, P. E., Soman, C., Wagner, M. R., Friesen, M. L., Kremer, J., Bennett, A., et al. 2017. Research priorities for harnessing plant microbiomes in sustainable agriculture. PLoS Biol. 15:e2001793.

Castrillo, G., Teixeira, P. J. P. L., Paredes, S. H., Law, T. F., De Lorenzo, L., et al. 2017. Root microbiota drive direct integration of phosphate stress and immunity. Nature 543:513-518.

Chelius, M. K., and Triplett, E. W. 2001. The diversity of Archaea and Bacteria in association with the roots of Zea mays L. Microbiol. Ecol. 41:252-263.

Cordell, D., Drangert, J.-O., and White, S. 2009. The story of phosphorus: Global food security and food for thought. Glob. Environ. Change 19: 292-305.

Csardi, G., and Nepusz, T. 2006. The igraph software package for complex network research. InterJournal. Complex Syst. 1695:1-9.

Darriba, D., Taboada, G. L., Doallo, R., and Posada, D. 2012. jModelTest 2: More models, new heuristics and parallel computing. Nat. Methods 9:772.

Desirò, A., Hao, Z., Liber, J. A., Benucci, G. M. N., Lowry, D., Roberson, R., et al. 2018. Mycoplasma-related endobacteria within Mortierellomycotina fungi: Diversity, distribution and functional insights into their lifestyle. ISME J. 12:1743-1757.

Desirò, A., Salvioli, A., Ngonkeu, E. L., Mondo, S. J., Epis, S., Faccio, A., et al. 2014. Detection of a novel intracellular microbiome hosted in arbuscular mycorrhizal fungi. ISME J. 8:257-270.

Dodt, M., Roehr, J., Ahmed, R., and Dieterich, C. 2012. FLEXBAR-Flexible barcode and adapter processing for next-generation sequencing platforms. Biology 1:895-905.

Durán, P., Thiergart, T., Garrido-Oter, R., Agler, M., Kemen, E., Schulze-Lefert, P., et al. 2018. Microbial interkingdom interactions in roots promote Arabidopsis survival. Cell 175:P973-983.e14.

Edgar, R. C. 2013. UPARSE: Highly accurate OTU sequences from microbial amplicon reads. Nat. Methods 10:996-998.

Fabiańska, I., Gerlach, N., Almario, J., and Bucher, M. 2018. Plant-mediated effects of soil phosphorus on the root-associated fungal microbiota in Arabidopsis thaliana. New Phytol. 435:824.

Flowers, J. J., He, S., Malfatti, S., del Rio, T. G., Tringe, S. G., Hugenholtz, P., et al. 2013. Comparative genomics of two "Candidatus Accumulibacter" clades performing biological phosphorus removal. ISME J. 7:2301-2314.

Gardes, M., and Bruns, T. D. 1993. ITS primers with enhanced specificity for basidiomycetes-application to the identification of mycorrhizae and rusts. Mol. Ecol. 2:113-118.

Gomes, E. A., Lana, U. G. P., Quensen, J. F., de Sousa, S. M., Oliveira, C. A., Guo, J., et al. 2018. Root-associated microbiome of maize genotypes with contrasting phosphorus use efficiency. Phytobiomes J. 2:129-137.
Gyaneshwar, P., Kumar, G. N., Parekh, L. J., and Poole, P. S. 2002. Role of soil microorganisms in improving $\mathrm{P}$ nutrition of plants. Plant Soil 245: 83-93.

Hamiaux, C., Drummond, R. S., Janssen, B. J., Ledger, S. E., Cooney, J. M., Newcomb, R. D., et al. 2012. DAD2 is an alpha/beta hydrolase likely to be involved in the perception of the plant branching hormone, strigolactone. Curr. Biol. 22:2032-2036.

Hartman, K., van der Heijden, M. G. A., Roussely-Provent, V., Walser, J.-C., and Schlaeppi, K. 2017. Deciphering composition and function of the root microbiome of a legume plant. Microbiome 5:2.

Hartman, K., van der Heijden, M. G. A., Wittwer, R. A., Banerjee, S., Walser, J.-C., and Schlaeppi, K. 2018. Cropping practices manipulate abundance patterns of root and soil microbiome members paving the way to smart farming. Microbiome 6:14.

Hassani, M. A., Durán, P., and Hacquard, S. 2018. Microbial interactions within the plant holobiont. Microbiome 6:58.

Hiruma, K., Gerlach, N., Sacristán, S., Nakano, R. T., Hacquard, S., Kracher, B., et al. 2016. Root endophyte Colletotrichum tofieldiae confers plant fitness benefits that are phosphate status dependent. Cell 165:464-474.

Huang, J., Hu, B., Qi, K., Chen, W., Pang, X., Bao, W., et al. 2016. Effects of phosphorus addition on soil microbial biomass and community composition in a subalpine spruce plantation. Eur. J. Soil Biol. 72:35-41.

Ihrmark, K., Bödeker, I. T. M., Cruz-Martinez, K., Friberg, H., Kubartova, A., Schenck, J., et al. 2012. New primers to amplify the fungal ITS2 region-evaluation by 454-sequencing of artificial and natural communities. FEMS Microbiol. Ecol. 82:666-677.

Ikoyi, I., Fowler, A., and Schmalenberger, A. 2018. One-time phosphate fertilizer application to grassland columns modifies the soil microbiota and limits its role in ecosystem services. Sci. Total Environ. 630:849-858.

Jacoby, R., Peukert, M., Succurro, A., Koprivova, A., and Kopriva, S. 2017. The role of soil microorganisms in plant mineral nutrition-current knowledge and future directions. Front. Plant Sci. 8:1617.

Jakobsen, I., Abbott, L. K., and Robson, A. D. 1992. External hyphae of vesicular-arbuscular mycorrhizal fungi associated with Trifolium subterraneum L. New Phytol. 120:509-516.

Jost, L. 2007. Partitioning diversity into independent alpha and beta components. Ecology 88:2427-2439.

Katoh, K., and Standley, D. M. 2013. MAFFT Multiple Sequence Alignment Software Version 7: Improvements in performance and usability. Mol. Biol. Evol. 30:772-780.

Lay, C.-Y., Hamel, C., and St-Arnaud, M. 2018. Taxonomy and pathogenicity of Olpidium brassicae and its allied species. Fungal Biol. 122:837-846.

Leff, J. W., Jones, S. E., Prober, S. M., Barberán, A., Borer, E. T., Firn, J. L., et al. 2015. Consistent responses of soil microbial communities to elevated nutrient inputs in grasslands across the globe. Proc. Natl. Acad. Sci. USA 112: 10967-10972

Liu, G., Pfeifer, J., de Brito Francisco, R., Emonet, A., Stirnemann, M., Gübeli, C., et al. 2018. Changes in the allocation of endogenous strigolactone improve plant biomass production on phosphate-poor soils. New Phytol. 217:784-798.

Magoč, T., and Salzberg, S. L. 2011. FLASH: fast length adjustment of short reads to improve genome assemblies. Bioinformatics 27:2957-2963.

Martin, M. (2011). Cutadapt removes adapter sequences from high-throughput sequencing reads. EMBnet.journal 17:10-12.

McGonigle, T. P., Miller, M. H., Evans, D. G., Fairchild, G. L., and Swan, J. A. 1990. A new method which gives an objective measure of colonization of roots by vesicular-arbuscular mycorrhizal fungi. New Phytol. 115:495-501.

McGuire, K. L., Payne, S. G., Palmer, M. I., Gillikin, C. M., Keefe, D., Kim, S. J., et al. 2013. Digging the New York City skyline: Soil fungal communities in green roofs and city parks. PLoS One 8:e58020.

McMurdie, P. J., and Holmes, S. 2013. phyloseq: An R package for reproducible interactive analysis and graphics of microbiome census data. PLoS One 8: e61217.

Oksanen, J., Blanchet, F. G., Friendly, M., Kindt, R., Legendre, P., McGlinn, D., et al. 2018. vegan: Community Ecology Package. http://ww25.cran.rproject.org/ packages/vegan/index.html

Öpik, M., Metsis, M., Daniell, T. J., Zobel, M., and Moora, M. 2009. Largescale parallel 454 sequencing reveals host ecological group specificity of arbuscular mycorrhizal fungi in a boreonemoral forest. New Phytol. 184: 424-437.

Quast, C., Pruesse, E., Yilmaz, P., Gerken, J., Schweer, T., Yarza, P., et al. 2013. The SILVA ribosomal RNA gene database project: Improved data processing and web-based tools. Nucleic Acids Res. 41:D590-D596.

R Core Team. 2016. R: A Language and Environment for Statistical Computing. R Core Team, Vienna, Austria. 
Rao, N. N., and Kornberg, A. 1996. Inorganic polyphosphate supports resistance and survival of stationary-phase Escherichia coli. J. Bacteriol. 178: 1394-1400.

Reddy, D. M. R. S., Schorderet, M., Feller, U., and Reinhardt, D. 2007. A petunia mutant affected in intracellular accommodation and morphogenesis of arbuscular mycorrhizal fungi. Plant J. 51:739-750.

Reijnders, L. 2014. Phosphorus resources, their depletion and conservation, a review. Resour. Conserv. Recycling 93:32-49.

Richner, W., and Sinaj, S. 2017. Grundlagen für die Düngung landwirtschaftlicher Kulturen in der Schweiz. Agroscope, Agrarforschung Schweiz.

Robbins, C., Thiergart, T., Hacquard, S., Garrido-Oter, R., Gans, W., Peiter, E., et al. 2018. Root-associated bacterial and fungal community profiles of Arabidopsis thaliana are robust across contrasting soil P levels. Phytobiomes J. 2:24-34.

Robinson, M. D., McCarthy, D. J., and Smyth, G. K. 2009. edgeR: A Bioconductor package for differential expression analysis of digital gene expression data. Bioinformatics 26:139-140.

Robinson, M. D., and Oshlack, A. 2010. A scaling normalization method for differential expression analysis of RNA-seq data. Genome Biol. 11:R25.

Rodríguez, H., and Fraga, R. 1999. Phosphate solubilizing bacteria and their role in plant growth promotion. Biotechnol. Adv. 17:319-339.

Ronquist, F., Teslenko, M., van der Mark, P., Ayres, D. L., Darling, A., Höhna, S., et al. 2012. MrBayes 3.2: Efficient Bayesian phylogenetic inference and model choice across a large model space. Syst. Biol. 61:539-542.

RStudio Team. 2015. RStudio: Integrated Development Environment for R. RStudio Team, Boston, MA.

Salvioli, A., Ghignone, S., Novero, M., Navazio, L., Venice, F., Bagnaresi, P., et al. 2016. Symbiosis with an endobacterium increases the fitness of a mycorrhizal fungus, raising its bioenergetic potential. ISME J. 10:130-144.

Schlaeppi, K., Bender, S. F., Mascher, F., Russo, G., Patrignani, A., Camenzind, T., et al. 2016. High-resolution community profiling of arbuscular mycorrhizal fungi. New Phytol. 212:780-791.

Schlaeppi, K., and Bulgarelli, D. 2015. The plant microbiome at work. Mol. Plant-Microbe Interact. 28:212-217.

Schloss, P. D., Westcott, S. L., Ryabin, T., Hall, J. R., Hartmann, M., Hollister, E. B., et al. 2009. Introducing mothur: Open-source, platform-independent, community-supported software for describing and comparing microbial communities. Appl. Environ. Microbiol. 75:7537-7541.

Schmieder, R., and Edwards, R. 2011. Quality control and preprocessing of metagenomic datasets. Bioinformatics 27:863-864.

Scholz, R. W., and Wellmer, F.-W. 2013. Approaching a dynamic view on the availability of mineral resources: What we may learn from the case of phosphorus? Glob. Environ. Change 23:11-27.

Sharma, S. B., Sayyed, R. Z., Trivedi, M. H., and Gobi, T. A. 2013. Phosphate solubilizing microbes: Sustainable approach for managing phosphorus deficiency in agricultural soils. Springerplus 2:587.

Sheldon, A. L. 1969. Equitability indices: Dependence on the species count. Ecology 50:466-467.

Silva, U. C., Medeiros, J. D., Leite, L. R., Morais, D. K., Cuadros-Orellana, S., Oliveira, C. A., et al. 2017. Long-term rock phosphate fertilization impacts the microbial communities of maize rhizosphere. Front. Microbiol. 8:1266.

Smith, S. E., and Read, D. 2008. Mycorrhizal Symbiosis, 3rd ed. Academic Press, London, U.K.
Spatafora, J. W., Chang, Y., Benny, G. L., Lazarus, K., Smith, M. E., Berbee, M. L., et al. 2017. A phylum-level phylogenetic classification of zygomycete fungi based on genome-scale data. Mycologia 108:1028-1046.

Stamatakis, A. 2014. RAxML version 8: A tool for phylogenetic analysis and post-analysis of large phylogenies. Bioinformatics 30:1312-1313.

Taktek, S., Trépanier, M., Servin, P. M., St-Arnaud, M., Piché, Y., Fortin, J. A., et al. 2015. Trapping of phosphate solubilizing bacteria on hyphae of the arbuscular mycorrhizal fungus Rhizophagus irregularis DAOM 197198. Soil Biol. Biochem. 90:1-9.

Tedersoo, L., Liiv, I., Kivistik, P. A., Anslan, S., Kõljalg, U., and Bahram, M. 2016. Genomics and metagenomics technologies to recover ribosomal DNA and single-copy genes from old fruit-body and ectomycorrhiza specimens. MycoKeys 13:1-20.

Tedersoo, L., Tooming Klunderud, A., and Anslan, S. 2018. PacBio metabarcoding of fungi and other eukaryotes: Errors, biases and perspectives. New Phytol. 217:1370-1385.

Tkacz, A., Cheema, J., Chandra, G., Grant, A., and Poole, P. S. 2015. Stability and succession of the rhizosphere microbiota depends upon plant type and soil composition. ISME J. 9:2349-2359.

Uehling, J., Gryganskyi, A., Hameed, K., Tschaplinski, T., Misztal, P. K., Wu, S., et al. 2017. Comparative genomics of Mortierella elongata and its bacterial endosymbiont Mycoavidus cysteinexigens. Environ. Microbiol. 19: 2964-2983.

van der Heijden, M. G. A., Dombrowski, N., and Schlaeppi, K. 2017. Continuum of root-fungal symbioses for plant nutrition. Proc. Natl. Acad. Sci. USA 114: 11574-11576.

Vandenbussche, M., Chambrier, P., Rodrigues Bento, S., and Morel, P. 2016. Petunia, your next supermodel? Front. Plant Sci. 7:577.

Vandenkoornhuyse, P., Quaiser, A., Duhamel, M., Le Van, A., and Dufresne, A. 2015. The importance of the microbiome of the plant holobiont. New Phytol. 206:1196-1206.

VDLUFA-Verlag (ed.) 2006. "Bestimmung von ausgewählten Elementen in pflanzlichem Material und Futtermitteln mit ICP-OES," in VDLUFA: Die chemische Untersuchung von Futtermitteln, Darmstadt.

Vierheilig, H., Coughlan, A., Wyss, U., and Piche, Y. 1998. Ink and vinegar, a simple staining technique for arbuscular-mycorrhizal fungi. Appl. Environ. Microbiol. 64:5004-5007.

Wakelin, S., Mander, C., Gerard, E., Jansa, J., Erb, A., Young, S., et al. 2012. Response of soil microbial communities to contrasted histories of phosphorus fertilisation in pastures. Appl. Soil Ecol. 61:40-48.

Wegmüller, S., Svistoonoff, S., Reinhardt, D., Stuurman, J., Amrhein, N., and Bucher, M. 2008. A transgenic dTph1 insertional mutagenesis system for forward genetics in mycorrhizal phosphate transport of Petunia. Plant J. 54: 1115-1127.

White, T. J., Bruns, T., Lee, S., and Taylor, J. W. 1990. Amplification and direct sequencing of fungal ribosomal RNA genes for phylogenetics. Pages 315-322 in: PCR Protocols: A Guide to Methods and Applications, Academic Press.

Yadav, V., Kumar, M., Deep, D. K., Kumar, H., Sharma, R., Tripathi, T., et al. 2010. A phosphate transporter from the root endophytic fungus Piriformospora indica plays a role in the phosphate transport to the host plant. J. Biol. Chem. 285:26532-26544.

Yao, Q., Li, Z., Song, Y., Wright, S. J., Guo, X., Tringe, S. G., et al. 2018. Community proteogenomics reveals the systemic impact of phosphorus availability on microbial functions in tropical soil. Nat. Ecol. Evol. 2:499-509. 